\title{
Erratum to: $\alpha$-Tocopheryl succinate causes mitochondrial permeabilization by preferential formation of Bak channels
}

\author{
Lubomir Prochazka $\cdot$ Lan-Feng Dong • \\ Karel Valis · Ruth Freeman · Stephen J. Ralph • \\ Jaroslav Turanek $\cdot$ Jiri Neuzil
}

Published online: 22 October 2013

(C) Springer Science+Business Media New York 2013

Erratum to: Apoptosis (2010) 15:782-794

DOI 10.1007/s10495-010-0482-z

There were some errors in band labeling in Figs. 3c, 7c and 9a that occurred during the assembly of the individual figures. The correct bands and labeling are shown in the figures below. The corrected western blots do not change nor affect the scientific meaning of the article. The authors apologize for this oversight.

The online version of the original article can be found under doi:10.1007/s10495-010-0482-z.

L. Prochazka $(\bowtie) \cdot$ J. Turanek

Veterinary Research Institute, Hudcova 70, 62100 Brno,

Czech Republic

e-mail: lubomir.pr@gmail.com

L.-F. Dong · R. Freeman · J. Neuzil ( $\square)$

Apoptosis Research Group, School of Medical Science and the Griffith Institute of Health and Medical Research,

Griffith University, Parklands Avenue, Southport, QLD 4222,

Australia

e-mail: j.neuzil@griffith.edu.au

K. Valis · J. Neuzil

Molecular Therapy Group, Institute of Biotechnology,

Czech Academy of Sciences, Prague, Czech Republic

\section{S. J. Ralph}

Genomic Research Centre, School of Medical Science,

Griffith University, Southport, QLD, Australia 

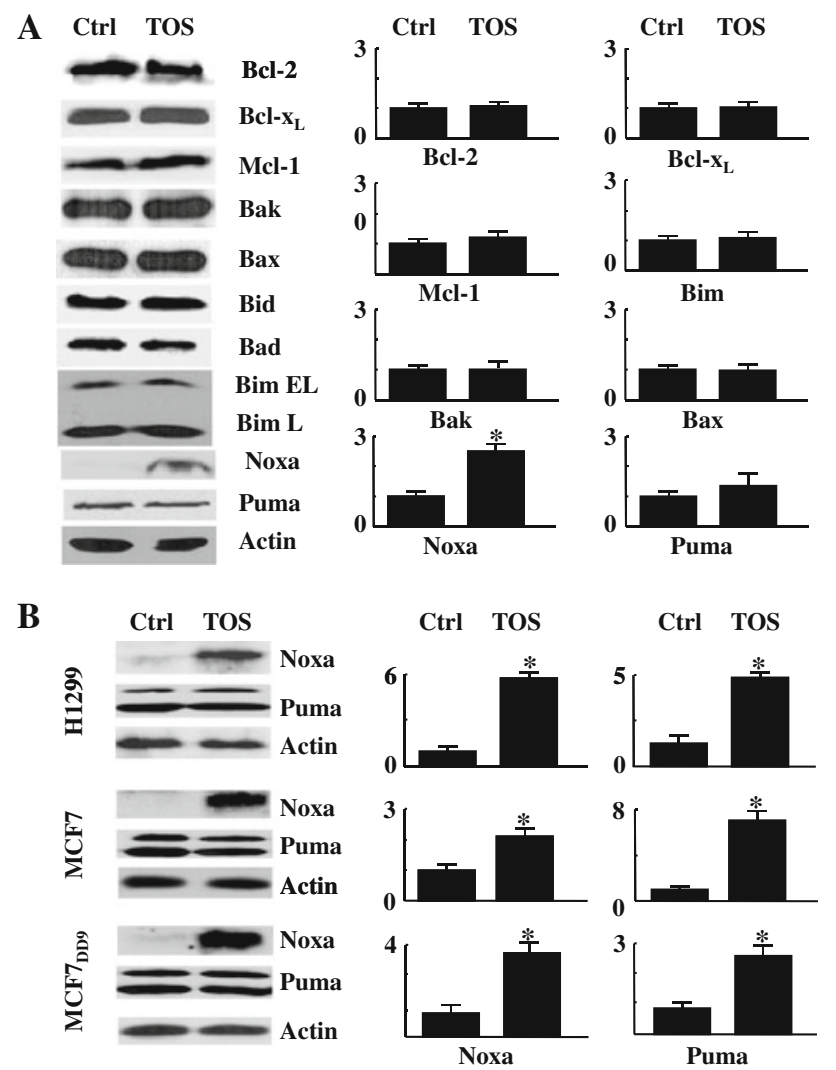

Puma

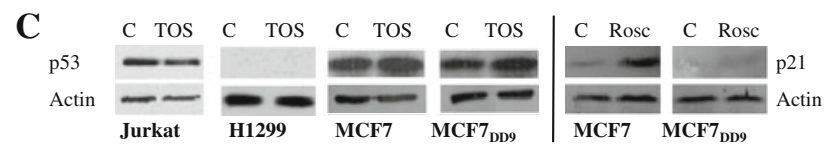

Fig. $3 \alpha$-TOS enhances expression of the BH3-only protein Noxa independently of cellular p53 status. Jurkat T lymphoma a and other cell lines b represented by MCF7 with wild-type p53, p53-deficient $\mathrm{H} 1299$ cells and MCF7 ${ }_{\text {DD } 9}$ cells with transcriptionally silent p53 were treated with $\alpha$-TOS (see below) and assessed for the expression of Bcl-2 family proteins a or only Noxa and Puma b using Q-PCR and Western blotting. Jurkat cells were exposed to $50 \mu \mathrm{M} \alpha$-TOS for $6 \mathrm{~h}$, H1299 cells to $80 \mu \mathrm{M} \alpha$-TOS for $12 \mathrm{~h}$, and MCF7 and MCF7 DD9 cells to $50 \mu \mathrm{M} \alpha$-TOS for $15 \mathrm{~h}$. $\mathbf{c}$ The control and $\alpha$-TOS-treated cells were also probed by western blotting for $\mathrm{p} 53$ or $\mathrm{p} 21$ protein levels in the case of MCF7 wt and MCF7DD9 cells and the cells were treated with $20 \mu \mathrm{M}$ Roscovitine for $20 \mathrm{~h}$ as a control for p53 dependent upregulation of $\mathrm{p} 21$ protein. Actin was used as a marker of equal protein loading for western blotting and as a house-keeping gene for Q-PCR. The Q-PCR data represent mean $\pm \operatorname{SEM}(n=3)$. The symbol ' $*$ ' denotes significant differences between control and treated cells with $P<0.05$. Western blot images are representative of at least three independent experiments
$\mathbf{A}$
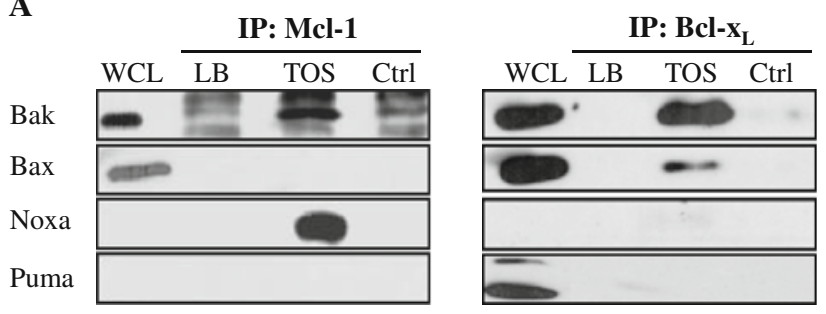$$
\text { B }
$$

B

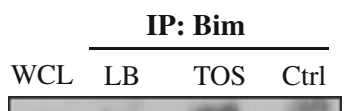

C Input

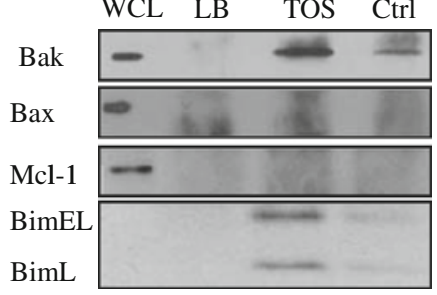

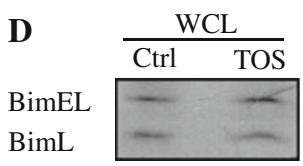

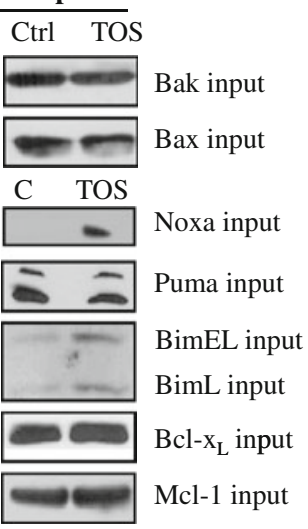

Fig. 7 Immunoprecipitation of selected $\mathrm{Bcl}-2$ family members confirms $\alpha$-TOS induced interactions among the Bcl-2 family. Mitochondrial lysates of H1299 cells were analyzed by antibody pull-down and western blotting as shown. Ctrl: non-treated cells; TOS: $\alpha$-TOS-treated cell samples with the antibodies as indicated. LB: immunoprecipitation using lysis buffer as a control for antibody cross-reactivity. WCL: whole cell lysates as positive control for Bak, Bax or other proteins as indicated. a Right hand panel shows proteins co-immunoprecipitating with Mcl-1. The left hand panel shows proteins co-immunoprecipitating with $\mathrm{Bcl}-\mathrm{x}_{\mathrm{L}}$. b Only Bak protein co-immunoprecipitated with Bim in mitochondrial lysates from $\alpha$-TOS treated cells. $\mathbf{c}$ The levels of protein expressed in samples of whole mitochondrial lysates. d Comparison of levels of Bim protein expressed in whole cell lysates from $\alpha$-TOS treated versus non-treated cells 
A

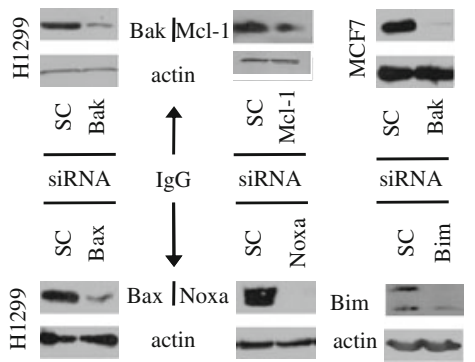

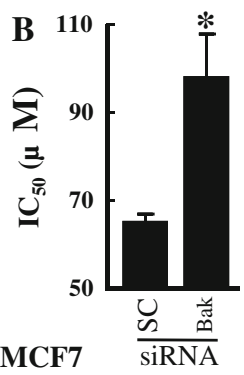

MCF7

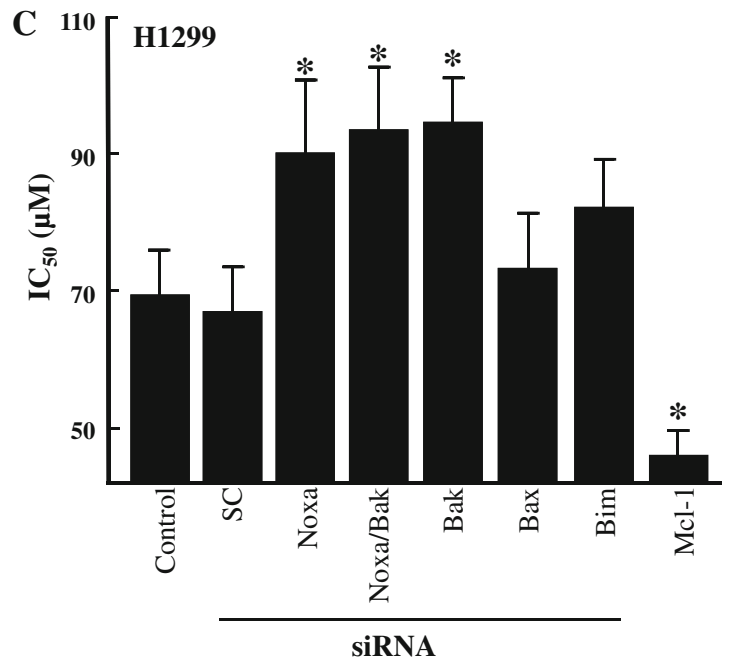

Fig. 9 Knocking down Noxa and Bak desensitizes cancer cells to $\alpha$-TOS-induced apoptosis. a Efficacy of downregulation of the Bak, Bax, Bim, Noxa and Mcl-1 proteins in H1299 and MCF7 cells using siRNA knock down. Actin was used as a standard for protein loading. b Change in the $\mathrm{IC}_{50}$ value for $\alpha$-TOS of MCF7 cells pre-treated with scrambled (SC) siRNA or Bak siRNA. c Changes in the $\mathrm{IC}_{50}$ value for $\alpha$-TOS of H1299 control cells or cells pre-treated with scrambled (SC), Noxa, Bak, Bax, Bim or Mcl-1 siRNA, or with Bak plus Noxa siRNA. Data show the mean $\pm 95 \%$ confidence interval, and the symbol ' $*$ ' denotes statistically significant differences from control cells with $P<0.05$ 